\title{
The role of flagella and chemotaxis genes in host pathogen interaction of the host adapted Salmonella enterica serovar Dublin compared to the broad host range serovar S. Typhimurium
}

\author{
John Elmerdahl Olsen ${ }^{1 *}$, Kirsten Hobolt Hoegh-Andersen ${ }^{1}$, Josep Casadesús ${ }^{2}$, Jesper T Rosenkrantz'
} Mark Simon Chadfield ${ }^{1,3}$ and Line Elnif Thomsen ${ }^{1}$

\begin{abstract}
Background: The importance of flagella and chemotaxis genes in host pathogen interaction in Salmonella enterica is mainly based on studies of the broad host range serovar, $S$. Typhimurium, while little is known on the importance in host specific and host adapted serovars, such as S. Dublin. In the current study we have used previously characterized insertion mutants in flagella and chemotaxis genes to investigate this and possible differences in the importance between the two serovars.

Results: fliC (encoding the structural protein of the flagella) was essential for adhesion and fliC and cheB (CheB restores the chemotaxis system to pre-stimulus conformation) were essential for invasion of S. Dublin into epithelial Int407 cells. In S. Typhimurium, both lack of flagella (fliC/fljB double mutant) and cheB influenced adhesion, and invasion was influenced by lack of both cheA (the histidine-kinase of the chemotaxis system), flic/fljB and cheB mutation. Uptake in J774A.1 macrophage cells was significantly reduced in cheA, cheB and fliC mutants of S. Dublin, while cheA was dispensable in S. Typhimurium. Removal of flagella in both serotypes caused an increased ability to propagate intracellular in $\mathbf{5 7 7 4}$ macrophage cells and decreased cytotoxicity toward these cells. Flagella and chemotaxis genes were found not to influence the oxidative response. The induction of IL-6 from J774A-1 cells depended on the presence of flagella in S. Typhimurium, whilst this was not the case following challenge with S. Dublin. Addition of flic from S. Typhimurium in trans to a flic mutant of $S$. Dublin increased cytotoxicity but it did not increase the IL-6 production. Flagella were demonstrated to contribute to the outcome of infection following oral challenge of mice in S. Dublin, while an S. Typhimurium fliC/fljB mutant showed increased virulence following intra peritoneal challenge.
\end{abstract}

Conclusions: The results showed that flagella and chemotaxis genes differed in their role in host pathogen interaction between S. Dublin and S. Typhimurium. Notably, lack of flagella conferred a more virulent phenotype in S. Typhimurium at systemic sites, while this was not the case in S. Dublin. In vitro assays suggested that this could be related to flagella-induced induction of the IL-6 pro-inflammatory response, but further in vivo studies are needed to confirm this.

\footnotetext{
* Correspondence: jeo@sund.ku.dk

${ }^{1}$ Department of Veterinary Disease Biology, Faculty of Health and Medical Sciences, University of Copenhagen, Stigbøjlen 4, 1870 Frederiksberg C, Denmark

Full list of author information is available at the end of the article
} 


\section{Background}

The flagellum of Salmonella enterica is made up of a single protein, flagellin, which consists of approximately 490 amino acids, and which differs between serovars [1]. For example fliC of $S$. Dublin and $S$. Typhimurium shows $38 \%$ identity at the DNA-level (BLASTN 2.2.1, NCBI) and 54\% identity at the amino acid level. Salmonella consist of more than 2500 serovars, most of which have two flagellin genes, $f l i C$ and $f l j B$, allowing antigen alteration [2]. The latter has been lost by secondary deletion in some lineages [3], for example $S$. Dublin only expresses flagellin encoded by fliC. A recent review suggests an evolutionary model, where fliC is the original and preferred gene, and $f l j B$ is only used under particular environmental conditions [3]. Flagella confer the ability of the bacterium to swim in liquid media. Chemical information received at membranereceptors influence the rotation of the flagellum motor, thus enabling the bacteria to respond to changes in the external environment by ordered motility. This signal transduction happens through the chemotaxis system (reviewed by Kojima and Blair [4]).

Flagella are recognized as PAMPs (pathogen associated molecular patterns) used by the host to recognize bacteria and besides their function in motility, flagella of $S$. Typhimurium have been shown to stimulate both the innate and adaptive immune system. Extracellular flagella activate toll-like receptor 5 (TLR-5) leading to a pro-inflammatory response with induction of cytokines (reviewed by Kawai and Akira [5]). Soluble flagellin in the cytosol induces pyroptotic cell death (see review by Miao et al. [6]) in a caspase-1-dependent manner through activation of the Nod like receptor NLRC4. This is in particular relevant in relation to intracellular bacteria, such as Salmonella, and a strain of $S$. Typhimurium that was manipulated to be unable to down regulate $f l i C$ expression intracellular was demonstrated to be attenuated during systemic infection [7].

Conflicting results have been reported on the importance of chemotaxis, flagellation and motility in host pathogen interaction in Salmonella. Flagella were found to be important for $S$. Typhimurium invasion of MODE$\mathrm{K}$ and Henle-407 cells, also when centrifugation was applied to maximize bacteria-to-cell contact. Hence the effect was considered unrelated to motility [8]. At the same time point, mutation of $\mathrm{fliC}$ and mutation of the motor protein $\operatorname{mot} A$ did not to influence intracellular cell numbers of $S$. Enteritidis in CaCo-2 cells [9]. This may, however, be a strain or cell specific response, since mutants of another $S$. Enteritidis strain showed reduced invasion in both Hep-2 and Div-1 cells [10]. Early challenge experiments using $\mathrm{C} 57 \mathrm{BL} / 6 \mathrm{~J}$ mice reported that flagella, whether functional or not, were virulence factors in $S$. Typhimurium, while chemotaxis genes were dispensable [11]. However, subsequent studies, with other strains have not been able to confirm the flagella phenotype $[8,12]$. Flagella but not fimbriae and not motility were found to be essential for $S$. Enteritidis infections in chicken [13], and lack of flagella causes a disadvantage in the early stage of oral infection of rats and in cell culture invasion $[14,15]$.

Salmonella serovars have very different epidemiology and life style, just as they display obvious differences with regard to motility and chemotaxis. The commonly studied $S$. Typhimurium infects numerous hosts and displays phase variation of its flagella antigens. The hostspecific and host-adapted serovars, on the other hand, infect a single or few hosts, and do not rely on extraanimal survival to any great extend [16]. It may be that motility and chemotaxis play a different role during host pathogen interaction in different serovars, depending on their lifestyle.

The current understanding of the importance of flagella and chemotaxis genes in Salmonella host pathogen interaction is derived from studies of $S$. Typhimurium and $S$. Enteritidis, and results based on these serovars are taken as general for the genus. Since the lifestyle differs markedly between ubiquitous serovars and the hostspecific/host-adapted ones, we hypothesized that this may be a wrong assumption. In order to investigate this, we characterized the importance of chemotaxis and flagella genes for host pathogen interaction of the host-adapted serovar $S$. Dublin compared to the wellcharacterized serovar $S$. Typhimurium.

\section{Results}

\section{Interaction with epithelial cells}

Salmonella normally infects through the faecal oral route. Several studies have reported that flagella are important for the intestinal phase of infection, mostly based on studies of the initial contact between cultured cells and flagella and motility mutants [8,17]. In this study we compared the adhesion and invasion of a wild type strain of $S$. Dublin to the smooth swimming cheA mutant, the tumbling cheB mutant and a mutant without flagella (fliC mutant). The corresponding mutants of $S$. Typhimurium were used as reference points. The results are shown in Table 1 . The $S$. Dublin flagella mutant $(\mathrm{fliC})$ was significantly reduced in adhesion and invasion, the constitutively tumbling $c h e B$ mutant was reduced in invasion, while the constitutively smooth swimming (cheA mutation) only showed a slight, non-significant reduction of adhesion and invasion. As can be seen from the Table 1, the flagella phenotype paralleled that of the flagella-less $S$. Typhimurium mutant, while cheA-mutation caused significantly reduced invasion and $c h e B-m u-$ tation both reduced adhesion and invasion in this serotype. 
Table 1 Adhesion and invasion of S. Dublin (SDu) and S. Typhimurium (STm) WT and flagella and chemotaxis mutants ${ }^{a}$ in cultured epithelial Int407 cells

\begin{tabular}{lll}
\hline Strain & Adhesion (\% of wild type) & Invasion (\% of wild type) \\
\hline SDu cheA & $47.8 \pm 5.6$ & $83.4 \pm 8.0$ \\
SDu cheB & $19.5 \pm 7.8$ & $2.4 \pm 0.9^{* * *}$ \\
SDu fliC & $6.0 \pm 3.3^{* * *}$ & $1.0 \pm 0.3^{* * *}$ \\
STm cheA & $76.2 \pm 33.5$ & $40.8 \pm 10.9^{* *}$ \\
STm cheB & $15.6 \pm 2.7^{* * *}$ & $1.2 \pm 1.3^{* * *}$ \\
STm fliC/fljB & $12.5 \pm 1.9^{* * *}$ & $0.4 \pm 0.3^{* * *}$ \\
\hline
\end{tabular}

a: Performance of mutant strains was compared statistically to the wild type strain of the same serovar. ${ }^{* *}: p<0.01 ; * * *: p<0.001$.

The inoculum of each strain was between $\log _{10} 7.9$ and $\log _{10} 8.2$ with no significant difference between strains.

\section{Uptake and survival inside macrophages}

Once Salmonella has invaded the host, professional phagocytic cells quickly take up the bacteria. Especially the uptake by macrophages has been considered important, deduced from the fact that all $S$. Typhimurium mutants that are attenuated for macrophage survival have turned out to be non-virulent in challenge experiments [18]. To investigate whether macrophage interaction depended on the presence of flagella and chemotaxis genes, we conducted experiments with cultured J774A.1 cells. The results are shown in Table 2. S. Dublin strains with mutation in $c h e A, c h e B$ and $f l i C$ were taken up by macrophages in significantly lower numbers than the wild type strain. The mutants of $S$. Typhimurium were found to have the same general uptake phenotypes, however, the differences between the wild type strain and the cheA mutant were not significant. All strains increased in numbers from 3 to 24 hours, but due to relatively large standard deviations, only the difference in net growth of the $S$. Typhimurium $f l i C / f l j B$ mutant was statistically different from that of the wild type strain. At 48 hours, wild type and chemotaxis mutants decreased in numbers, however, the $c h e B$ mutant of $S$. Typhimurium was significantly less reduced compared to the wild type strain. Contrary to this, flagella-less mutants of both serotypes showed net growth, but only the $S$. Typhimurium strains was significantly different from the wild type strains.

\section{Cytotoxicity towards macrophage cell line J774A.1}

Results of the macrophage assays above may be influenced by cytotoxicity of the strain, since strains that kill the macrophages subject themselves to the action of the antibiotic gentamicin in the culture medium. A comparison of cytotoxicity towards the J774A.1 cells after 24 hours is shown in Figure 1. The non-flagellated mutants of $S$. Dublin and $S$. Typhimurium were less cytotoxic than the wild type strains, in line with previous observations that flagella influence Salmonella induction of macrophage cell death [19]. The net growth of flagella mutants in the survival assays above could thus be a result of decreased killing of macrophages. The chemotaxis mutants of $S$. Dublin did not differ significantly from the wild type strain, while the cheA mutant of $S$. Typhimurium was slightly, but significantly, less cytotoxic than the wild type strain.

Wild type $S$. Dublin was less cytotoxic than wild type $S$. Typhimurium (Figure 1). To investigate whether this was related to the flagella type, we provided the $\mathrm{fliC} \mathrm{mu}-$ tant of $S$. Dublin with $S$. Typhimurium fliC in trans on the plasmid pPR2. The fliC mutant itself was negative with $\mathrm{H}: p, g$ (S. Dublin flagella antigen) and $\mathrm{H}: i, \mathrm{H}: 2$ (S. Typhimurium flagella antigen) by serotyping and Western blot, while the complemented strain was positive with $\mathrm{H}: i$ and $\mathrm{H}: 2$ typing sera. It was non-motile but expressed a high number of flagella as demonstrated by electron microscopy (data not shown). It did not differ significantly from the wild type strain in interactions with epithelial cells or macrophages (data not shown).

Table 2 Uptake and survival of S. Dublin 3246 (SDu) and S. Typhimurium (STm) wildtype and flagella and chemotaxis mutants in cultured J774A.1 macrophages ${ }^{\mathrm{a}}$

\begin{tabular}{|c|c|c|c|}
\hline Strain & $\begin{array}{l}\text { Uptake } 3 \mathrm{~h} \\
\text { (Percent of wild type strain) }\end{array}$ & $\begin{array}{l}\text { Survival } 24 \mathrm{~h} \\
\text { (Percent of same strain at } 3 \mathrm{~h} \text { ) }\end{array}$ & $\begin{array}{l}\text { Survival } 48 \mathrm{~h} \\
\text { (Percent of same strain at } 3 \mathrm{~h} \text { ) }\end{array}$ \\
\hline SDu WT & 100 & $124,1 \pm 43.5$ & $20.7 \pm 4.7$ \\
\hline SDu cheA & $53.9 \pm 15.1^{* *}$ & $279.8 \pm 65.8$ & $53.8 \pm 16.5$ \\
\hline SDu cheB & $1.4 \pm 1.0^{* *}$ & $307.7 \pm 90.2$ & $248.8 \pm 39.8$ \\
\hline SDu flic & $1.0 \pm 0.2^{* * *}$ & $450.5 \pm 255.0$ & $615.3 \pm 325.8$ \\
\hline STm WT & 100 & $114.0 \pm 42.6$ & $2.8 \pm 1.72 .8$ \\
\hline STm cheA & $72.4 \pm 22.4$ & $100.2 \pm 31.0$ & 12.2. \pm 3.1 \\
\hline STm cheB & $19.0 \pm 9.3^{* *}$ & $309.8 \pm 231.5$ & $81.7 \pm 6.9^{*}$ \\
\hline STm fliC/flijB & $0.2 \pm 0.1^{* * *}$ & $490.9 \pm 111.6^{*}$ & $702.9 \pm 53.0^{* * *}$ \\
\hline
\end{tabular}

a: Uptake of mutant strains was expressed relatively to and compared statistically to the wild type strain of the same serovar. Survival at 24 and 48 hours was expressed relatively to the number of bacteria determined at 3 hours and compared statistically to the survival capability of the wild type strain of the same serotype. *: $p<0.05 ;{ }^{* *}: p<0.01{ }^{* * *}: p<0.001$. 


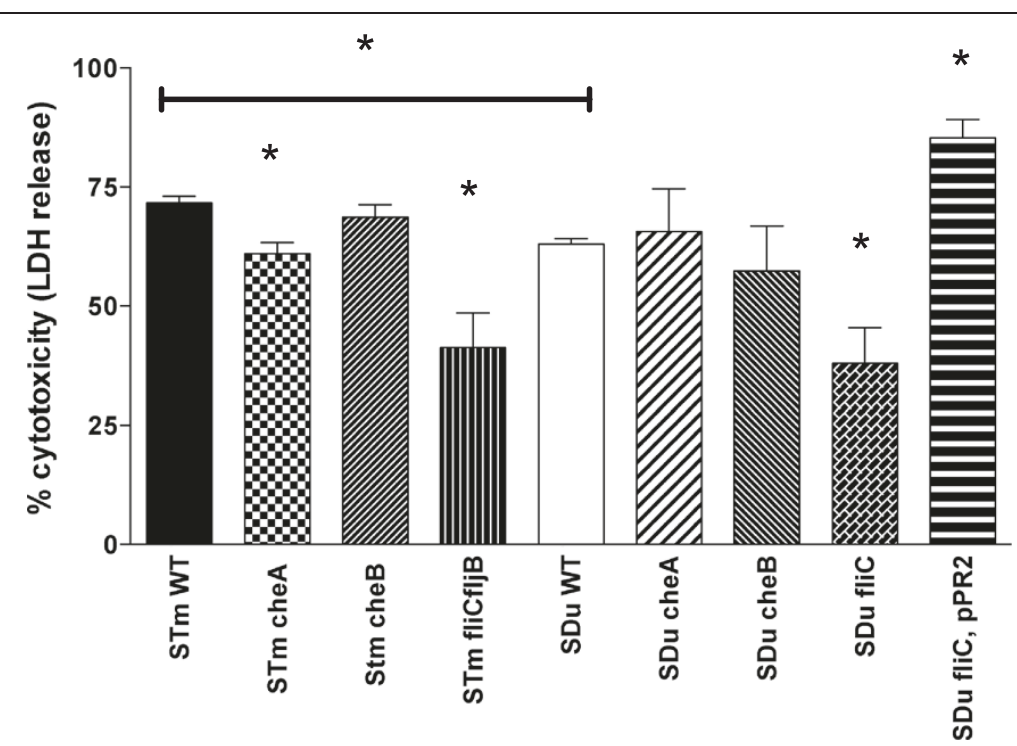

Figure 1 Cytotoxicity of strains of S. Dublin (SDu) and S. Typhimurium (STm) in J774A.1 macrophages. Cytotoxicity was measured 24 hours post challenge with flagellar (SDu fliC and STm flic/fljB) and chemotaxis mutants (cheA and cheB) and the wild type strains. Significant $(p<0.05)$ differences between wild type and mutant strains are shown with *. The cytotoxicity of the two wild type strains was also compared, and this was shown to be statistically different, as indicated by the * in the top of the figure.

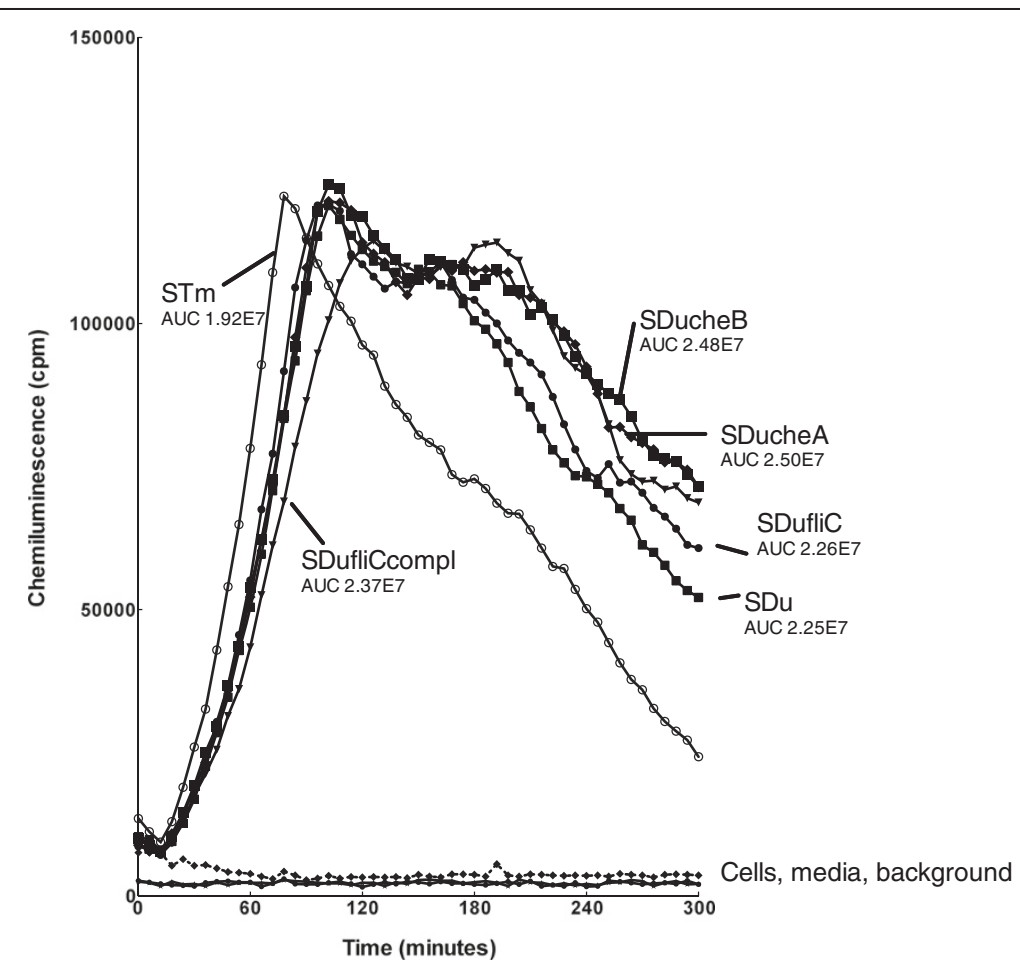

Figure 2 Oxidative responses of J774A.1 macrophages following challenge with wild type and chemotaxis and flagella mutant of S. Dublin (SDu) and S. Typhimurium (STm). The response is measured in arbitrary chemiluminescence units. Positive and negative controls are indicated. 
The complemented fliC mutant of $S$. Dublin was significantly more cytotoxic than the wild type strain of $S$. Dublin, above the level of the wild type strain of $S$. Typhimurium (Figure 1).

\section{The importance of chemotaxis and flagella genes for induction of oxidative burst in macrophages}

The ability of the strains to stimulate the oxidative burst in J774A.1 cells was investigated. Wild type strains differed in induction of oxidative response in the sense that the wild type strain of $S$. Typhimurium peaked early compared to the wild type strain of $S$. Dublin, and showed a significantly lower area under the response curve (AUC). Only relative small differences in the oxidative burst were observed between $S$. Dublin wild type and mutant strains, and none of the differences were statistically significant (Figure 2). Knock out of flagella and chemotaxis genes also did not influence the oxidative burst significantly in $S$. Typhimurium (data not shown). When the $S$. Dublin fliC mutant was complemented with $S$. Typhimurium $\mathrm{fliC}$, the response peaked later but the magnitude of response (AUC) was not affected (Figure 2).

\section{Induction of cytokines IL-6 response in cultured J774A.1 macrophages}

As mentioned in the introduction, flagellin has been reported to stimulate a pro-inflammatory response with induction of cytokines including IL-6 [5]. We wanted to investigate how the IL-6 response depended on the presence of flagella and chemotaxis genes. After 1 hour, no significant IL- 6 production was seen in any of the strains (data not shown), however, after 4 hours, strains of both serovars had induced a strong production of IL-6 (Figure 3). In $S$. Typhimurium, mutation in both flagella genes independently or together, as well as mutation of cheB, caused a reduced IL-6 response, while surprisingly, lack of flagella did not cause a reduction in $S$. Dublin. IL-6 levels following challenge of cells with ten times higher doses of $S$. Typhimurium fliCfljB and $S$. Dublin $\mathrm{fliC}$ mutants did not change the responses compared to the normal challenge dose. Complementation of $f l i C$ in $S$. Dublin with fliC from $S$. Typhimurium in trans caused a dramatic reduction of IL-6 from the infected macrophages.

\section{Oral and intra peritoneal challenge of mice}

The chemotaxis mutants did not differ significantly from the wild type strains following oral challenge. The $S$. Dublin $\mathrm{fliC}$ mutant showed lower CFU in the spleen 4-5 days post challenge (CI: $0.46(\mathrm{p}<0.01))$, while the $S$. Typhimurium $f l i C / f l j B$ mutant did not differ markedly from the wild type strain (CI: 1.12), however, the difference was statistically significant. Lack of flagella has

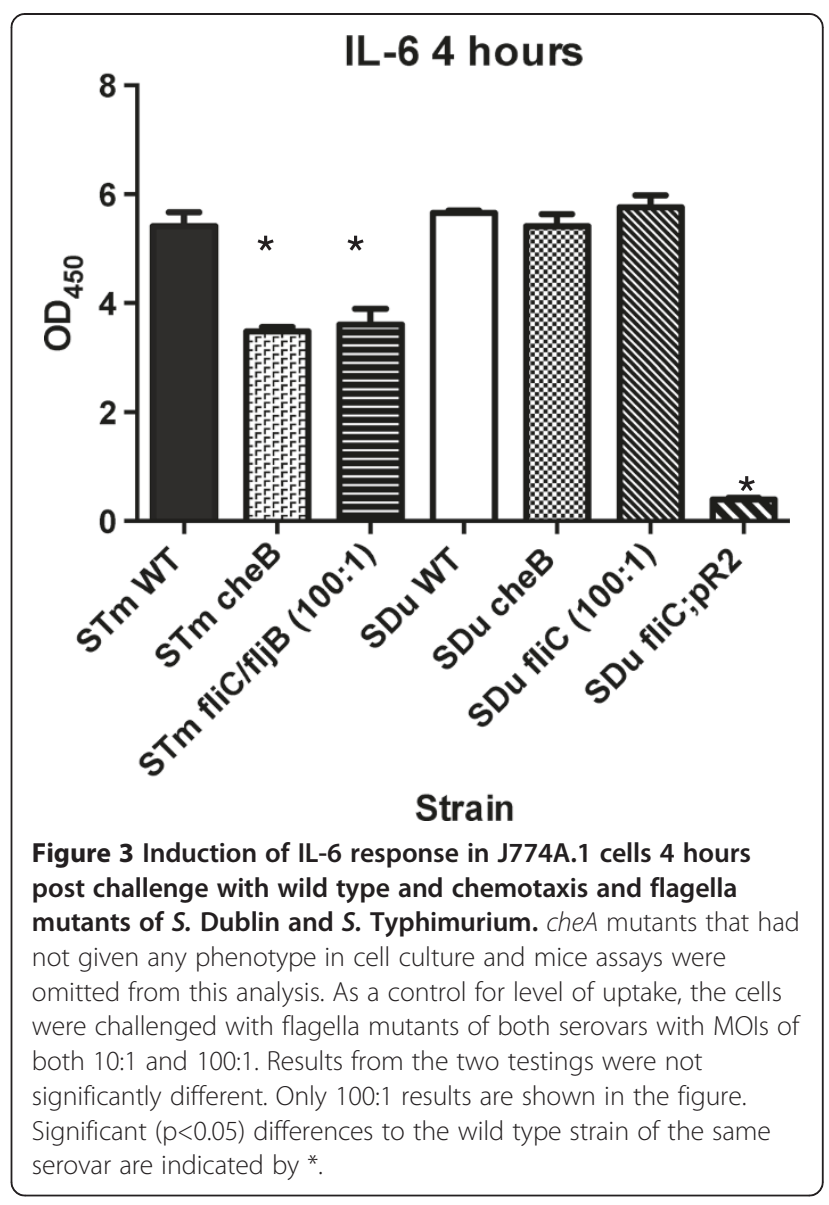

been reported to increase fitness of $S$. Typhimurium during systemic infection of mice [8]. We therefore also investigated the importance of flagella genes using intra peritoneal challenge, thereby bypassing the intestine. The $S$. Typhimurium $f l i C / f l j B$ mutant showed increased numbers of bacteria in the spleen (CI: 1.78; $\mathrm{p}<0.0001$ ), which corroborates previous studies [8]. The corresponding flagella-less $S$. Dublin mutant did not show this phenotype (CI: 0.91) (Table 3).

\section{Discussion}

In the current study we used chemotaxis and flagella mutants of the host adapted serovar $S$. Dublin and corresponding mutants of the broad host range serovar $S$. Typhimurium to study possible serovar differences in the importance of these genes for host pathogen interaction. The studies were based on defined mutants in one strain of each serovar, and we cannot rule out that there may be strain differences within serovar.

The constitutively tumbling cheB S. Dublin mutant, but not the constitutively smooth swimming cheA mutant, was negatively affected in invasion of epithelial cells. Since cheA has previously been shown to be important for $S$. Typhimurium cell invasion [20], which we 
Table 3 Virulence phenotypes of flagella and chemotaxis mutants of S. Dublin (SDu) and S. Typhimurium (STm) in C57/B6 mice

\begin{tabular}{llll}
\hline Mutant & Challenge route $^{\mathbf{a}}$ & $\mathbf{C l}^{\mathbf{b}}$ S.Du & $\mathbf{C l}^{\mathbf{b}} \mathbf{S T m}$ \\
\hline cheA & p.o. & 1.03 & 1.09 \\
cheB & p.o. & 0.97 & 1.05 \\
fliC & p.o. & $0.46^{* *}$ & - \\
fliC & i.p. & 0.91 & - \\
flic/fljB & p.o. & - & $1.12^{* *}$ \\
fliC/fljB & i.p. & - & $1.78^{* * *}$ \\
\hline
\end{tabular}

a: p.o. $=$ per oral challenge; i.p. $=$ intraperitoneal challenge .

b: The competitive index was calculated as the ratio of mutant to wild type in the spleen 4-5 days post infection divided by the ratio of mutants to wild type strain in the input pool. Indexes where the output was significantly different from the input pool are marked with ${ }^{* *}(p<0.01)$ and ${ }^{* *}(p<0.001)$.

also observed in our studies, $S$. Typhimurium and $S$. Dublin apparently differ with respect to the role of $c h e A$ in epithelial cell invasion. Lack of flagella (fliC mutation) caused reduced adhesion, which is in accordance with previously reported results for the effect of $f l i C / f l j B$ mutation in S. Typhimurium [17] and our observations on the role of flagella in this serotype.

It has previously been reported that it is the flagella and not motility, which are important for cell adhesion and invasion [17], but it is currently unknown how precisely flagella influence this in a motility independent way, at least in cell culture experiments. Since we used centrifugation to maximize cell contact, it is also unlikely that our results were caused by reduced motility, which would lead to a reduction in number of contacts between bacteria and cells. Flagella in $S$. Typhimurium are expressed inside epithelial cells and can be demonstrated in infected cultured HeLa cells [21]. During in vivo invasion, the stimulation of TLR- 5 by flagellin and the following pro-inflammatory response may be important. However, invasion by $S$. Typhimurium in cell culture experiments happens within 15 minutes [22], and it is unlikely to be influenced by secretion of stimulating factors. A more likely explanation is downregulation of SPI1 in flagella mutants, as suggested by Kim et al. [23]. This down regulation can be caused by several regulatory systems, which control both flagella and virulence gene expression [24,25].

Whether motility per se can be ruled out as important for invasion is still controversial since motility was shown to be essential for invasion of $S$. Typhi into cultured epithelial cells [26]. A recent study with $S$. Typhimurium also suggests a requirement for motility in infection of epithelial cells. The invading population was demonstrated to consist of two populations. Some cells were only infected with few bacteria, which did not multiply to any great extent. These bacteria showed down-regulation of SPI-1 and fliC transcription. A fraction of approximately $10 \%$ of cells, however, was infected with bacteria that were motile, expressed invasion genes, possessed flagella, and multiplied at high rate. A speculation is that these cells may be ready to reenter the lumen of the intestine to re-infect other cells [22]. Whether a similar picture can be seen for $S$. Dublin remains to be investigated.

Similar to invasion into epithelial cells, mutation of chemotaxis and flagella genes caused reduced uptake by macrophage cells. The reason for this is unknown. The flagella and chemotaxis genes are down regulated once S. Typhimurium is inside a macrophage [27], probably to prolong the time the bacterium can stay inside the macrophage protected from neutrophil killing in the extracellular environment [7]. The intracellular down regulation is controlled by the gene $y d i V$, which prevents transcription of the flagellin promoter [28]. It is currently unknown how $S$. Dublin regulates it flagella expression in response to macrophage uptake. Despite the down regulation, flagella of $S$. Typhimurium are important for the outcome of the systemic phase of an infection, since lack of flagella leads to a decrease in the percentage of CD14+ and CD54+ cells resulting in a reduction of uptake of soluble antigens by these cells and fewer bacteria accumulating intracellular [29,30]. Flagellin induces $\mathrm{I}-\kappa \mathrm{B} \alpha$ degradation and subsequent NF- $\mathrm{kB}$ nuclear translocation, and induction of nitric oxide synthase [31-33]. This induces rapid de novo synthesis of tumour necrosis factor alpha (TNF- $\alpha$ ), interferon gamma (IFN- $\gamma$ ), interleukin- $1 \beta$ (IL-1 $\beta$ ) followed by IL- 6 and IL-10, which is typical for a systemic inflammatory response.

Lack of flagella was found to allow net growth inside the macrophages over a 48 hours period, while wild type and chemotaxis mutant strains were reduced in numbers. The SPI-1 encoded type three-secretion system and flagella are important for rapid host cell death by pyroptosis seen after cell infection with $S$. Typhimurium [19]. In the present investigation, lack of flagella caused reduced extracellular levels of lactate dehydrogenase, the intracellular enzyme used as an indicator of macrophage cell death, and this reduced killing can be the reason for the net growth observed with flagella-less mutants. The present investigation does not allow us to conclude which underlying mechanism that was responsible for the reduced cell death when flagella were absent. Wild type $S$. Typhimurium was significantly more cytotoxic than $S$. Dublin. When $S$. Dublin expressed S. Typhimurium $\mathrm{fliC}$, the cytotoxicity increased above $S$. Typhimurium levels. This indicates that $f l i C$ is important for the level of cytotoxicity, however, the complemented strain used to show this had a higher number of flagella than the wild type strain, and we cannot rule out that this causes the increase in cytotoxicity. The plasmid used for complementation was based on pMF3, which 
has previously been used to complement knock out phenotypes in $S$. Typhimurium without adverse effects [34]. More detailed studies are needed to demonstrate how these serotype differences relate to differences in the flagella sequence.

Significant cytokine production is generally assumed to require phagocytosis of the bacteria [35]. This corresponds to uptake in our assays, and as pointed out by Winther et al. [36] knock out mutants are not well suited to distinguish between lack-of-stimulation and lack-of-internalization responses. The flagella mutant of $S$. Typhimurium caused a reduced IL-6 cytokine production, but it also showed reduced uptake. We therefore included a control experiment where a 10 times higher challenge dose of the flagella mutant was used. The high challenge dose did not increase the IL- 6 production, indicating that the lack of response was most likely not related to invasion levels. In support of this conclusion, the $f l i C$ and $c h e B$ mutants of $S$. Dublin also showed significantly reduced invasion, but absence of these genes in $S$. Dublin did not influence cytokine production. This result point to a fundamental difference between $S$. Dublin and $S$. Typhimurium in the way the flagella stimulates the host response, and calls for more detailed studies on structural functional relations in the signalling to the host.

The $S$. Dublin $f l i C$ mutant with $S$. Typhimurium provided in trans induced a lower response than the wild type strain. This result was surprising. Its phenotype is similar to a motA mutation, i.e. structurally the flagella appears normal, but they do not move. Naturally occurring $\operatorname{mot} A$ mutants of $S$. Enteritidis stimulated transcriptional pro-inflammatory responses in Caco-2 cells [37], and there is no obvious reason why the complemented $S$. Dublin strain should not do the same. In cell culture experiment, a motA mutant of $S$. Typhimurium was non-invasive [19], which differs from the phenotype of our complemented mutant, and further studies are needed to clarify this observation.

Lack of stimulation of IL-6 expression has previously been seen with the host-specific serovar $S$. Gallinarum in a comparison to $S$. Typhimurium and $S$. Enteritidis after infection of a primary chicken cell line [38]. No control was included in that study for the fact that $S$. Gallinarum contrary to $S$. Typhimurium and $S$. Enteritidis lacks flagella. Our results indicate that lack of IL-6 induction may be a general feature of host adapted/ host specific serotypes. Host specific serovar has been speculated to perform stealth like infection (i.e. down regulates several host responses) in comparison to the ubiquitous serovars [39]. The lower cytotoxicity and lack of IL-6 responses support this assumption. In contrast to the role in IL-6 induction, none of the mutants differed significantly from the wild type strains in induction of oxidative responses. This result suggested that flagellin was not important for induction of the oxidative response.

Results on the role of flagella and chemotaxis genes in Salmonella host pathogen interaction have been contradictory (compare [12] and [8] with [11]), and we purposely looked for a sensitive assay to show subtle differences between strains. Co-infection assays have been shown to be more sensitive than assays where strains are tested individually [40]. Using this assay, we found that flagella significantly influenced the number of bacteria that could be isolated from the spleen 4-5 days post oral infection of mice with $S$. Dublin, but not with $S$. Typhimurium. Chemotaxis genes were found to be dispensable in this assay, as previously reported for $S$. Typhimurium [11]. Animal welfare regulations dictated us to scarify mice when they were severely affected by infection, and this prevented us from using one single end-point of infection. Potentially, this may have influenced the competitive indexes for $S$. Typhimurium, since this serovar propagated at different speed at systemic sites depending on the presence of flagella genes (see below). However, all mice were killed within a 24 hours period, and we do not believe that this significantly influenced our results.

Like cheA mutation, mutation of cheR confers a constitutively smooth swimming phenotype. We have not included this gene in our investigation, and we cannot rule out that it may have a different role in host pathogen interaction than cheA. We have performed preliminary testing of an $S$. Dublin cheR mutant and found that it corresponds to $c h e A$ with respect to phenotypes in cell assays and oral challenge of mice (unpublished), however, we do not have $S$. Typhimurium results to compare it to.

Flagella have been found to be important for the outcome of oral infection with $S$. Typhimurium in streptomycin treated mice, which is a model for studies of the entero-pahtogenesis of Salmonella [41]. In this model flagella are essential for initiation of inflammation, creating an environment in which Salmonella prevails over the normal flora, and in this model, chemotaxis genes were also essential for the outcome of infection. Cattle are the natural host for $S$. Dublin, and in addition to differences caused by the choice of animal model, studies have shown that virulence factors may differ depending on the host [42]. This must be taken into account when concluding on the current results. The changes in virulence observed when flagella were removed were relatively modest. We have previously demonstrated that flagella do not play an important role during extra animal survival [43], and we believe that selection for stable maintenance of the flagella apparatus must happen during the interaction with the host. It thus appears that these small differences are enough to provide the selective force. 
It has previously been reported that a flagella mutant of $S$. Typhimurium is hyper virulent following intraperitoneal challenge of mice [8] and we confirmed this result. In contrast, the $S$. Dublin flagella mutant was not different from the wild type strain after intraperitoneal challenge. In conjunction with the results of IL-6 induction and cytotoxicity, this indicates that flagella are most important for $S$. Dublin in the initial invasion phase in the intestine, while it plays a minor role during the systemic phase. We suggest that a likely explanation for the contradicting results on the role of flagella in virulence of $S$. Typhimurium is that the results depends very much on the time point where bacterial load is measured. At early time points, lack of flagella causes a lower invasion, but at later time points, this is balanced by a higher ability to grow in the systemic phase.

\section{Conclusion}

The results show that flagella but not chemotaxis genes influence the outcome of $S$. Dublin infection following oral challenge in the mouse model, and that $S$. Dublin flagella do not appear to be important during the systemic phase of infection. This points to fundamental differences in bacteria host signalling between Salmonella serotypes, and shows that results from studies of $S$. Typhimurium cannot be assumed to be general to the genus.

\section{Methods}

\section{Strains and growth conditions}

Well characterized flagella and chemotaxis insertion mutants of S. Dublin 3246 and S. Typhimurium 4/74 (Table 4) were obtained from a previous study [43]. The pMF3 derived plasmid pPR2 (TH2422) encoding $S$. Typhimurium $f l i C$ was kindly provided by Dr. Kelly $\mathrm{T}$. Hughes, Washington University, Seattle, USA and was used to provide this gene in trans to $S$. Dublin. Plasmid extraction was performed with the QIAgen purification kit, as described by the manufacturer and electroporation was carried out as described by Maloy et al. [44].

Unless otherwise stated, strains were cultured in LB broth (Difco) overnight at $37^{\circ} \mathrm{C}$. Stock cultures were maintained frozen at $-80^{\circ} \mathrm{C}$ in $\mathrm{LB}$ supplemented with glycerol $(33 \% \mathrm{w} / \mathrm{v})$. To prepare inoculate for in vivo and in vitro studies, stocks were thawed and inoculated on LB-agar containing 1,5\% agar (Difco) and plates were incubated overnight at $37^{\circ} \mathrm{C}$. Single representative colonies were inoculated into fresh LB broth and incubated overnight at $37^{\circ} \mathrm{C}$. Media were supplemented with relevant antibiotics (Sigma) at concentrations: kanamycin $(50 \mu \mathrm{g} /$ $\mathrm{ml})$, tetracycline $(20 \mu \mathrm{g} / \mathrm{ml})$, ampicillin $(50 \mu \mathrm{g} / \mathrm{ml})$ and chloramphenicol $(20 \mu \mathrm{g} / \mathrm{ml})$.

\section{Motility measurement}

Motility was assayed in Heart Infusion broth with $0.25 \%$ agar (Difco) and on Swarm agar (Statens Serum Institute, DK) as described [43].

\section{Expression of flagella antigens}

Serotyping was performed as previously described [43]. Western blot was performed using NuPAGE ${ }^{\text {Tx }} 12,5 \%$ Tris- $\mathrm{HCl}$ gels (Novex) as instructed by the manufacturer and specific flagella antisera (H:i, $\mathrm{H}: 2$ or $\mathrm{H}: \mathrm{p}, \mathrm{g})$, (Statens Serum Institute (SSI), Denmark).

\section{Demonstration of flagella by electron microscopy}

To demonstrate flagella, bacteria were negatively stained with uranyl acetate $2 \%$ and examined by transmission electron microscopy at an instrumental magnification of 27500.

\section{Adhesion and survival properties in vitro}

Comparison of in vitro adhesion, invasion (uptake) and survival of bacteria inside cells was performed using the epithelial cell line Int407 and the macrophage-like cell line, J774A.1, as previously described [46]. Before experiments with macrophages, bacteria were opsonised with

Table 4 Bacterial strains and their motility phenotypes

\begin{tabular}{|c|c|c|c|}
\hline Strain & Description; Relevant genotype & Motility phenotype & Source \\
\hline JEO 3774 & Wild-type Salmonella Typhimurium 4/74 & Wild type & [45] \\
\hline JEO 3665 & Wild-type Salmonella Dublin 3246 & Wild type & [45] \\
\hline JEO880 & JEO 3774 (cheA::Tn10a) & Smooth & [43] \\
\hline JEO881 & JEO 3774 (cheB::Tn10a) & Tumbling & [43] \\
\hline JEO885 & JEO 3774 (fliC::MudJ; fljB::MudJCme) & None & [43] \\
\hline JEO886 & JEO 3665 (flic::Mud」 ${ }^{\mathrm{b}}$ ) & None & [43] \\
\hline JEO887 & JEO 3665 (flic::MudJ; pPR2 ${ }^{d}$ ) & None & This study \\
\hline JEO888 & JEO 3665 (cheA::Tn10a) & Smooth & [43] \\
\hline JEO889 & JEO 3665 (cheB::Tn10a) & Tumbling & [43] \\
\hline
\end{tabular}

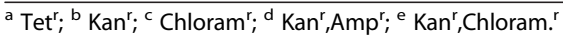


$10 \%$ heat treated foetal calf serum (Invitrogen) for 30 min at $37^{\circ} \mathrm{C}$ prior to addition to the cells. Infections were performed at a multiplicity of infection (m.o.i.) of 10:1 with the macrophage cell line and 100:1 with Int 407 cells. For all experiments, cells were centrifuged at $1500 \mathrm{rpm}$ for $2 \mathrm{~min}$. immediately after infection to allow close contact of the bacteria with the cells. Each bacterial strain was assayed in triplicate and experiments were repeated once.

\section{Cytotoxicity}

Cytotoxicity to macrophages was determined by release of lactate dehydrogenase (LDH) by the monolayers into supernatants using the CytoTox $96^{\circledR}$ Non-Radioactive Cytotoxicity Assay (Promega G1780). Results were expressed as the percentage of LDH released by infected monolayers compared to LDH release by lysis buffer treated (lysed) monolayers at 24 hours [ $\left(\mathrm{A}_{495}\right.$ test sample - $\mathrm{A}_{495}$ medium control) / ( $\mathrm{A}_{495}$ macrophage+lysis buffer - $\mathrm{A}_{495}$ medium+lysis buffer)] $\times 100$.

\section{Induction of oxidative radicals (chemiluminescence)}

The method described by Chadfield and Olsen [47] was used. Opsonized zymosan (Sigma) and phorbol myristate acetate (PMA)(Sigma) was used as positive control stimuli. A Lucigenin probe (Sigma) dissolved in DMSO (Sigma) and diluted in Hanks balanced salt solution (HBBS) (Gibco Life Technologies) to final assay concentrations of $150 \mu \mathrm{g} / \mathrm{ml}$ was used. Cells used in the assays were J774A.1. The luminometer (AUTOLUMAT LB 953, Berthold) was set at $37^{\circ} \mathrm{C}$. The reading intervals were minutes and the duration of the assays were 300 minutes. The response was expressed as the area under the response curve (AUC) and the time where the response peaked.

\section{Induction of IL-6 production}

A macrophage invasion assay was conducted with J774A.1. After 1 hour and 4 hours of incubation, the last 3 hours with gentamicin present in the medium, supernatants were removed and assayed for the presence of cytokine IL-6 using a commercially available kit (Promokine, mouse IL-6 ELISA kit). Positive controls consisted of purified IL-6 supplied with the kit, and negative controls consisted of wells not infected with bacteria.

\section{Animal challenge experiments}

Per oral and intraperitoneal virulence were assessed using competitive challenge assays with five C57BL/6 female mice (Taconic Black6 mice) of 6-8 weeks of age per group. The protocol followed the instructions of Jelsbak et al. [48] for intra peritoneal challenge, while a challenge dose of $8 \times 10^{6} \mathrm{CFU}$ was used for per oral challenges. In all experiments, $S$. Dublin was given a 10 times reduced dose compared to $S$. Typhimurium. The ratio between the wild type and the mutant strain in the broth used for challenge as well as the ratio in the spleen 4-5 days post challenge was determined by patching of 100 colonies from the broth and from the spleen of each mice onto LB agar without antibiotic and 100 colonies onto LB agar with the relevant antibiotic. For statistical analysis of the difference between input and output ratios, an estimate of the variation on the input ratio was needed. This was obtained by combining the results from the patching of all input pools into one distribution and using this as an average input ratio. The animal experimentation was conducted with permission from the Animal Experiments Inspectorate (http://www.foedevarestyrelsen. dk/Dyr/Dyrevelfaerd/Dyreforsoegstilsynet/Sider/forside.aspx) in accordance with Danish law (license number: 2009/ 561-1675).

\section{Statistical analysis}

Statistical analyses were made using the statistical software package GraphPath Prism 5. Mean CFU of bacterial strains in cell assays and cytotoxicity levels were compared using Bonferroni's multiple comparison test. Comparison of mean competitive index between wild type and mutant strains and oxidative responses were done using unpaired T-test. $\mathrm{P}<0.05$ was considered significant.

\section{Abbreviations}

$\mathrm{Cl}$ : Competitive index; AUC: Area under curve; CFU: Colony forming units.

\section{Competing interests}

The authors declare that they have no competing interests.

\section{Authors' contributions}

$\mathrm{KH}-\mathrm{H}-\mathrm{A}$ and JC constructed the strains, $\mathrm{KH}-\mathrm{HA}, \mathrm{MSC}$ and JEO conducted cell culture adhesion and invasion experiments, MSC measured the oxidative response in macrophages, JEO measured cytokine responses, $\mathrm{KH}-\mathrm{HA}$, JEO and JR conducted mice challenge experiments, JEO drafted the manuscript and all authors read and commented on this. All authors approved the final manuscript.

\section{Acknowledgments}

Tony Bønnelycke and Gitte Pedersen are thanked for skillful technical assistance. Kelly T. Hughes, Washington University, Seattle, WA is thanked for providing the plasmid pPR2 with S. Typhimurium fliC. José Breschiani is thanked for help with the electron-microscopy pictures.

\section{Author details}

${ }^{1}$ Department of Veterinary Disease Biology, Faculty of Health and Medical Sciences, University of Copenhagen, Stigbøjlen 4, 1870 Frederiksberg C, Denmark. 'Department of Genetics, Faculty of Biology, University of Seville, Apartado 1095, 41080 Sevilla, Spain. ${ }^{3}$ Novo Nordic, Krogshøjvej 51, 2880 Bagsværd, Denmark.

Received: 4 July 2012 Accepted: 19 March 2013

Published: 25 March 2013

\section{References}

1. Joys TM: The covalent structure of the phase-1 filament protein of Salmonella Typhimurium and its comparison with other flagellins. J Biol Chem 1985, 260:15758-15761.

2. Popoff MY: LL: Antigenic formulas of the Salmonella serovars. Paris: WHO collaboration Centre for reference and research on Salmonella; 2007. 
3. McQuiston JR, Fields PI, Tauxe RV, Logsdon JMJ: Do Salmonella carry spare tyres. Trends Microbiol 2008, 16:142-148.

4. Kojima S, SBlair DF: The bacterial flagellar motor: structure and function of a complex molecular machine. Int Rev Cyt 2004, 233:93-134.

5. Kawai T, Akira S: Toll-like receptors and their crosstak with other innate receptors in infection and immunity. Immune Int Rev Cytol 2011, 34:637-650

6. Miao EA, Rajan JV, Aderem A: Caspase-1-induced pyroptotic cell death. Immunol Rev 2011, 243:206-214.

7. Miao EA, Leaf IA, Treuting PM, Moa DP, Dors M, Sarkar A, Warren SE, Wewers MD, Aderem A: Caspase-1-induced pryoptosis is an innate immune effector mechanism against intracellular bacteria. Nat Immunol 2010, 11:1136-1143

8. Schmidt CK, Ikeda JS, Darnell SC, Watson PR, Bispham J, Wallis TS, Weinstein $\mathrm{DL}$, Metcalf ES, O'Brien AD: Absence of all components of the flagellar export and synthesis machinery differentially alters virulence of Salmonella enterica serovar Typhimurium in models of typhoid fever, survival in macrophages, tissue culture invasiveness, and calf enterocolitis. Infect Immune 2001, 69:5619-5625.

9. Van Asten FJ, Hendriks HG, Koninkx JF, Van der Zeijst BA, Gaastra W: Inactivation of the flagellin gene of Salmonella enterica serovar Enteritidis strongly reduces invasion into differentiated Caco-2 cells. FEMS Microbiol Lett 2000, 185:175-179.

10. La Ragione RM, Cooley WA, Velge P, Jepson MA, Woodward MJ: Membrane ruffling and invasion of human and avian cell lines is reduced for aflaggelate mutants of Salmonella enterica serovar Enteritidis. Int J Med Microbiol 2003, 293:261-272

11. Carsiotis M, Stocker BAD, Weinstein DL, O'Brien AD: Flagella of Salmonella typhimurium are a virulence factor in infected $\mathrm{C} 57 \mathrm{BL} / 6 \mathrm{~J}$ mice. Infect Immun 1984, 46:814-818.

12. Lockman HA, Curtiss R 3rd: Virulence of non-type 1-fimbriated and nonfimbriated nonflagellated Salmonella typhimurium mutants in murine typhoid fever. Infect Immun 1988, 60:491-496.

13. Allen-Vercoe E, Sayers AR, Woodward MJ: Virulence of Salmonella enterica serovar Enteritidis aflagellate and afimbriate mutants in a day-old chick model. Epidemiol Infect 1999, 122:395-402.

14. Robertson JM, Grant G, Allen-Vercoe E, Woodward MJ, Pusztai A, Flint HJ: Adhesion of Salmonella enterica serovar Enteritidis strains lacking fimbriae and flagella to rat ileal explants cultured at the air interface or submerged in tissue culture medium. J Med Microbiol 2000, 49:691-696.

15. Robertson JM, McKenzie NM, Duncan M, Allen-Vercoe E, Woodward MJ, Flint HJ, Grant G: Lack of flagella disadvantages Salmonella enterica serovar Enteritdis during the early stages of infection in the rat. $J$ Med Microbiol 2003, 52:91-99.

16. Uzzau S, Brown DJ, Wallis T, Rubino S, Leori G, Bernard S, Casadesús J, Platt DJ, Olsen JE: Host adapted serovars of Salmonella enterica. Epidemiol Infect 2000, 125:229-255.

17. Khoramian-Falsafi T, Harayama S, Kursukake K, Pechère JC: Effect of motility and chemotaxis on the invasion of Salmonella typhimurium into HeLa cells. Microbial Pathog 1990, 9:47-53.

18. Fields PI, Swanson RV, Hardaris CG, Heffron F: Mutants of Salmonella typhimurium that cannot survive within the macrophage are avirulent. Proc Nat Acad Sci, USA 1986, 83:5189-5193.

19. Fink SL, Cookson BT: Pyroptosis and host cell death responses during Salmonella infection. Cell Microbiol 2007, 9:2562-2570.

20. Jones BD, Lee CA, Falkow S: Invasion by Salmonella typhimurium is affected by the direction of flagellar rotation. Infect Immun 1992 60:2475-2480.

21. Hautefort I, Thompson A, Eriksson-Ygberg S, Parker ML, Lucchini S, Danino V, Bongaerts RJ, Ahmad N, Rhen M, Hinton JC: During infection of epithelial cells Salmonella enterica serovar Typhimurium undergoes a time-dependent transcriptional adaptation that results in simultaneous expression of three type 3 secretion systems. Cell Microbiol 2008, 10:958-984.

22. Knodler LA, Vallance BA, Celli J, Winfree S, Hansen B, Montero M, SteeleMortimer O: Dissemination of invasive Salmonella via bacterial-induced extrusion of mucosal epithelia. Proc Nat Acad Sci, USA 2010, 107:17733-17738.

23. Kim M, Lim S, Kim D, Choy HE, Ryu S: A tdcA mutation reduces the invasive ability of Salmonella enterica serovar Typhimurium. Mol Cells 2009, 28:89-395.
24. Mangan MW, Lucchini S, Croinin TO, Fitzgerald S, Hinton JCD, Dorman CJ: The nucleoid-associated protein $\mathrm{HU}$ controls three regulons that coordinate virulence, response to stress and general physiology in Salmonella enterica serovar Typhimurium. Microbiol 2011, 175:1075-1087.

25. Webber MA, Bailey AM, Blair JMA, Morgan E, Stevens MP, Hinton J, Ivens A, Wain J, Piddock LJV: The global consequence of disruption of the AcrABTolC efflux pump in Salmonella enterica includes reduced expression of SPI-1 and other attributes required to infect the host. J Bac 2009, 191:4276-4285.

26. Liu SL, Ezaki T, Miura H, Matsui K, Yabuuchi X: Intact motility as a Salmonella typhi invasion-related factor. Infect Immun 1988, 56:1967-1973.

27. Eriksson S, Lucchini S, Thompson A, Rhen M, Hinton JC: Unraveling the biology of macrophage infection by gene expression profiling of intracellular Salmonella enterica. Mol Microbiol 2003, 47:103-118.

28. Stewart MK, Cummings LA, Johnson ML, Berezow AB, Cookson BT: Regulation of phenotypic heterogeneity permits Salmonella evasion of he host caspase-1 inflammatory response. PNAS 2011, 108:20742-20747.

29. Wyant TL, Tanner MK, Sztein MB: Salmonella typhi flagella are potent inducers of proinflammatory cytokine secretion by human monocytes. Infect Immun 1999, 67:3619-3624.

30. Metcalfe HJ, Best A, Kanellos T, La Ragione RM, Werling D: Flagellin expression enhances Salmonella accumulation in TLR5-positive macrophages. Develop Compar Immunol 2010, 34:797-804.

31. Eaves-Pyles T, Murthy K, Liaudet L, Virag L, Ross G, Soriano FG, Szal C, Salzman AL: Flagellin, a novel mediator of Salmonella-induced epithelial activation and systemic inflammation: I kappa B alpha degradation, induction of nitric oxide synthase, induction of proinflammatory mediators, and cardiovascular dysfunction. J Immunol 2001, 166:1248-1260.

32. Gewirtz AT, Navas TA, Lyons S, Godowski PJ, Madara JL: Cutting edge: Bacterial flagellin activates basolaterally expressed tlr5 to induce epithelial proinflammatory gene expression. J Immunol 2001, 167:1882-1885.

33. Hayashi F, Smith KD, Ozinsky A, Hawn TR, Yi EC, Goodlett DR, Eng JK, Akira $S$, Underhill DM, Aderem A: The innate immune response to bacterial flagellin is mediated by Toll-like receptor 5. Nat 2001, 410:1099-1103.

34. Jones BD, Falkow S: Identification and characterization of a Salmonella typhimurium oxygen-regulated gene required for bacterial internalization. Infect Immune 1994, 62:37-45.

35. Yrlid U, Svensson M, Johansson C, Wick MJ: Salmonella infection of bone marrow-derived macrophages and dendritic cells: influence on antigen presentation and initiation of immune response. FEMS Immun Med Microbiol 2000, 27:313-320.

36. Winter SE, Thiennimitr $P$, Nuccio S-P, Haneda T, Winter MG, Wilson RP, Russel JM, Henry T, Tran QT, Lawhon SD, Adams LG, Bäumler AJ: Contribution of flagellin pattern recognition to intestinal inflammation during Salmonella enterica infection. Infect Immun 2009, 77:1904-1916.

37. Yim L, Betancor L, Martinez A, Bryant C, Maskell D, Chabalgoity JA: Naturally occurring motility-defective mutants of Salmonella enterica serovar Enteritidis isolated preferentially from nonhuman rather than human sources. Appl Environment Microbiol 2011, 77:7740-7748.

38. Kaiser P, Rothwell L, Galyov EE, Barrow PA, Burnside J, Wigley P: Differential cytokine expression in avian cells in response to invasion by Salmonella typhimurium, Salmonella enteritidis and Salmonella gallinarum. Microbiol 2000, 146:3217-3226.

39. Tsolis RM, Young GM, Solnick JV, Bäumler AJ: From bench to bedside: stealth of enteroinvasive pathogens. Nat Rev Microbio/ 2008, 6:883-892

40. Beuzón CR, Holden DW: Use of mixed infections with Salmonella strains to study virulence genes and their interactions in vivo. Microb Infect 2001, 3:1345-1352.

41. Stecher B, Hapfelmeier S, Müller C, Kremer M, Stallmach T, Hardt W-D: Flagella and chemotaxis are required for efficient induction of Salmonella enterica serovar Typhimurium colitis in the streptomycintreated mice. Infect Immun 2004, 72:4138-4150.

42. Pullinger GD, Dziva F, Charleston B, Wallis TS, Stevens MP: Identification of Salmonella enterica serovar Dublin specific sequences by subtractive hybridization and analysis of their role in intestinal colonization and systemic translocation in cattle. Infect Immun 2008, 76:5310-5321.

43. Olsen JE, Hoegh-Andersen KH, Casadesús J, Thomsen LE: The importance of motility and chemotaxis for extra animal survival of Salmonella 
enterica serovar Typhimurium and Dublin. J Appl Microbiol 2012, 113:560-568.

44. Maloy SR, Stewart VJ, Tayler RK: Genetic analysis of pathogenic bacteria. New York: Cold Spring Harbor Press; 1996.

45. Watson PR, Paulin SM, Bland AP, Jones PW, Wallis TS: Characterization of intestinal invasion by Salmonella Typhimurium and Salmonella Dublin and the effect of a mutation in the invH gene. Infect Immun 1995, 63:2743-2754.

46. Chadfield MS, Brown DJ, Aabo S, Christensen JP, Olsen JE: Comparison of intestinal invasion and macrophage response of Salmonella Gallinarum and other host adapted Salmonella enterica serovars in the avian host. Vet Microbiol 2003, 92:49-64.

47. Chadfield MS, Olsen JE: Determination of the oxidative burst chemiluminescent response of avian and murine-derived macrophages versus corresponding cell-lines in relation to stimulation with Salmonella serovars. Vet Immunol Immunopathol 2001, 80:289-308.

48. Jelsbak L, Thomsen LE, Wallrodt I, Jensen PR, Olsen JE: Polyamines are required for virulence in Salmonella enterica serovar Typhimurium. PLoS One 2012, 7:e36149.

doi:10.1186/1471-2180-13-67

Cite this article as: Olsen et al:: The role of flagella and chemotaxis genes in host pathogen interaction of the host adapted Salmonella enterica serovar Dublin compared to the broad host range serovar $S$. Typhimurium. BMC Microbiology 2013 13:67.

\section{Submit your next manuscript to BioMed Central and take full advantage of:}

- Convenient online submission

- Thorough peer review

- No space constraints or color figure charges

- Immediate publication on acceptance

- Inclusion in PubMed, CAS, Scopus and Google Scholar

- Research which is freely available for redistribution 